\title{
The perioperative outcomes of robot-assisted radical cystectomy with intracorporeal urinary diversion compared with laparoscopic radical cystectomy with extracorporeal urinary diversion at a single institution.
}

\author{
Atsuto Suzuki ( $\nabla$ satsuto0421@gmail.com ) \\ Yokohama City University Hospital \\ Kentaro Muraoka \\ Yokohama City University Hospital \\ Tomoyuki Tatenuma \\ Yokohama City University Hospital \\ Kimito Osaka \\ Kanagawa Prefectural Ashigarakami Hospital \\ Yumiko Yokomizo \\ Fujisawa Shonandai Hospital \\ Narihiko Hayashi \\ Yokohama City University Hospital \\ Hisashi Hasumi \\ Yokohama City University Hospital \\ Yusuke Ito \\ Yokohama City University Hospital \\ Keichi Kondo \\ Yokohama City University Hospital \\ Noboru Nakaigawa \\ Yokohama City University Hospital \\ Kazuhide Makiyama \\ Yokohama City University Hospital
}

\section{Research Article}

Keywords: bladder cancer, ileal conduit, intracorporeal urinary diversion, extracorporeal urinary diversion, robot-assisted radical cystectomy, perioperative outcome 
Posted Date: September 2nd, 2021

DOI: https://doi.org/10.21203/rs.3.rs-821069/v1

License: (c) (i) This work is licensed under a Creative Commons Attribution 4.0 International License. Read Full License 


\section{Abstract \\ Background}

The number of facilities adapting intracorporeal urinary diversion (ICUD) using robots instead of extracorporeal urinary diversion (ECUD) is increasing, but the clinical evidence is limited.

\section{Methods}

We retrospectively analyzed 26 consecutive patients who underwent ICUD with an ileal conduit following robot-assisted radical cystectomy (RARC) between 2018 and 2020 (RARC + ICUD group), and compared them with 26 consecutive patients who underwent ECUD with an ileal conduit following laparoscopic radical cystectomy (LRC) between 2012 and 2019 (LRC + ECUD group) at Yokohama City University.

\section{Results}

In both groups, the patient background was similar except for age and neoadjuvant chemotherapy. In the RARC + ICUD group vs. the LRC + ECUD group, the median total operation time was 516 min vs. 532.5 min $(p=0.217)$, the time to cystectomy was $163 \mathrm{~min}$ vs. $194.5 \mathrm{~min}(p=0.007)$, and the time of urinary diversion with an ileal conduit was $161 \mathrm{~min}$ vs. $201.5 \mathrm{~min}(\mathrm{p}<0.001)$. The postoperative maximum value of C-reactive protein was $6.98 \mathrm{mg} / \mathrm{L}$ vs. $12.46 \mathrm{mg} / \mathrm{L}(\mathrm{p}=0.001)$. The median number of days to oral intake was 3 days vs. 5 days ( $p=0.003$ ). The median length of hospital stay was 17 days vs. 32 days ( $<0.001$ ). The postoperative complication rate (within 90 days) was $23.1 \%$ vs. $42.3 \%(p=0.237)$. ClavienDindo classification $\geq 3$ was noted in 1 vs. 4 patients $(p=0.350)$. The median number of dissected lymph nodes was 20 vs. $15(p=0.008)$.

\section{Conclusions}

RARC + ICUD was superior to LRC + ECUD in terms of time to cystectomy and ileal conduit urinary diversion, invasiveness, and lymph node dissection. We consider ICUD with an ileal conduit following RARC to be the more advantageous procedure.

\section{Background}

Radical cystectomy is performed as the standard treatment for invasive bladder cancer.[1] However, open radical cystectomy (ORC) and urinary diversion using the gastrointestinal tract can cause many perioperative complications, being a highly invasive treatment.[2]

Therefore, in recent years, laparoscopic radical cystectomy (LRC) and robot-assisted laparoscopic radical cystectomy (RARC) have been performed to reduce the invasiveness.[3] 
Until recently, extracorporeal urinary diversion (ECUD) with laparoscopy was selected instead of intracorporeal urinary diversion (ICUD) with laparoscopy because of the complexity of the procedure, the extended operation time, gastrointestinal complications, and postoperative recovery delay. $[4,5]$

However, due to the gradual spread of robotic surgery, the number of facilities performing ICUD with robots is increasing as an alternative to ECUD due to the potential benefits of a smaller incision, reduced pain, less bowel exposure, and lower risk of fluid imbalance.[6] The number of studies evaluating the benefits of ICUD compared with ECUD is increasing, but no consensus has been reached.

This study compared the perioperative outcomes of ICUD after RARC with those of ECUD after LRC at a single institution.

\section{Methods}

Patients who underwent LRC or RARC for bladder cancer at our institution between February 2012 and September 2020 were analyzed. We retrospectively evaluated 26 consecutive patients who underwent ICUD after RARC between February 2018 and September 2020 (RARC + ICUD group) and compared them with 26 consecutive patients who underwent ECUD after laparoscopic radical cystectomy (LRC) between February 2012 and April 2019 at our hospital (LRC + ECUD group). After removal of the bladder, lymph node dissection was performed laparoscopically in both groups. In this study, common iliac lymph node dissection was defined as extended lymph node dissection. Following lymph node dissection, we carried out urinary diversion. The ileal conduit was mainly constructed using the Bricker method.[7, 8] In ECUD, we made a 4-5-cm incision for the ileal conduit. In general, RARC + ICUD and LRC + ECUD were performed or instructed by a single surgeon.

Complications were graded according to the modified Clavien-Dindo classification. If the patient had multiple complications, the highest Clavien-Dindo grade was adopted in this study.

The primary outcome was the postoperative complication rate in the RARC + ICUD group compared with that in the LRC + ECUD group. The measured perioperative outcomes included operative time, estimated blood loss, blood transfusion, intraoperative complications, lymph node dissection, pathological TN stage, positive surgical margin, days to walking, drinking and oral intake, length of hospital stay, postoperative maximum value of C-reactive protein, WBC and LDH, follow-up period, and postoperative complications. In both groups, we defined the time of urinary diversion as from the end of lymph node dissection to the end of the operation, which included ureter mobilization from the left to the right retroperitoneum, retroperitonealization of the ileal ureteral anastomosis, and stoma creation.

This study was approved by the institutional review board of Yokohama City University.

We used the t-test to compare the averages of continuous variables and chi-square tests to compare the proportions of categorical variables between the groups. The threshold for significance was $P<0.05$. 
All statistical analyses were performed using EZR (Saitama Medical Center, Jichi Medical University, Saitama, Japan), which is a graphical user interface for $\mathrm{R}$ (The R Foundation for Statistical Computing, Vienna, Austria). More precisely, it is a modified version of $\mathrm{R}$ commander designed to add statistical functions frequently used in biostatistics.[9]

\section{Results}

The patient background in both groups is shown in Table 1. The RARC + ICUD group included 24 men and 2 women. The LRC + ECUD group included 21 men and 5 women. The median age was significantly lower in the RARC + ICUD group than in the LRC + ECUD group. There were no differences in sex, body mass index (BMI), or clinical T or $\mathrm{N}$ stage. The RARC + ICUD group received significantly more neoadjuvant chemotherapy than the LRC + ECUD group $(21 / 26,80.8 \%$ vs. $12 / 26,46.1 \% ; p=0.001)$. 
Table 1

Patient Background

\begin{tabular}{|c|c|c|c|}
\hline & RARC + ICUD $(n=26)$ & LRC + ECUD $(n=26)$ & P-value \\
\hline Median age, years (IQR) & $69(55.00-71.75)$ & $70.5(66.25-75.50)$ & 0.041 \\
\hline Sex (\%) & & & 0.419 \\
\hline Male & $24(92.3)$ & $21(80.8)$ & \\
\hline Female & $2(7.7)$ & $5(19.2)$ & \\
\hline Median BMI (IQR) & $22.00(20.75-24.77)$ & $23.00(21.25-25.00)$ & 0.340 \\
\hline Clinical T stage (\%) & & & 0.135 \\
\hline $\mathrm{Ta}$, Tis & $1(3.8)$ & $0(0)$ & \\
\hline T1 & $4(15.4)$ & $3(11.5)$ & \\
\hline T2 & 5 (19.2) & $8(30.8)$ & \\
\hline T3 & $13(50.0)$ & $6(23.1)$ & \\
\hline $\mathrm{T} 4$ & $3(11.5)$ & $8(30.8)$ & \\
\hline Clinical N stage (\%) & & & 0.648 \\
\hline NO & $22(84.6)$ & $20(76.9)$ & \\
\hline N1 & $2(7.7)$ & $4(15.4)$ & \\
\hline N2 & $2(7.7)$ & $1(3.8)$ & \\
\hline N3 & $0(0)$ & $1(3.8)$ & \\
\hline Clinical M stage (\%) & & & 1 \\
\hline MO & $26(100.0)$ & $25(96.2)$ & \\
\hline M1 & 0 & $1(3.8)$ & \\
\hline Neoadjuvant chemotherapy (\%) & & & 0.001 \\
\hline None & $5(19.2)$ & $14(53.9)$ & \\
\hline \multicolumn{4}{|l|}{ IQR $=25 \%-75 \%$ interquartile range } \\
\hline \multicolumn{4}{|l|}{${ }^{\dagger} \mathrm{GC}=$ gemcitabine/cisplatin } \\
\hline \multicolumn{4}{|l|}{${ }^{{ }^{\mathrm{G}} \mathrm{G} \text { Carbo }=\text { gemcitabine/carboplatin }}$} \\
\hline \multicolumn{4}{|l|}{$\S_{G N}=$ gemcitabine $/$ nedaplatine } \\
\hline${ }^{9}$ CDDP/CPT-11 = cisplatin/irinot & & & \\
\hline
\end{tabular}




\begin{tabular}{|c|c|c|c|}
\hline & RARC + ICUD $(n=26)$ & LRC + ECUD $(n=26)$ & P-value \\
\hline $\mathrm{GC}^{\dagger}$ & $16(61.6)$ & $3(11.5)$ & \\
\hline GCarbo $^{\ddagger}$ & $3(11.5)$ & $0(0)$ & \\
\hline GN§ & $0(0)$ & $5(19.2)$ & \\
\hline CDDP/CPT-11 & $2(7.7)$ & $4(15.4)$ & \\
\hline \multicolumn{4}{|c|}{ IQR $=25 \%-75 \%$ interquartile range } \\
\hline \multicolumn{4}{|c|}{${ }^{\dagger} \mathrm{GC}=$ gemcitabine $/$ cisplatin } \\
\hline \multicolumn{4}{|c|}{${ }^{\ddagger}$ GCarbo = gemcitabine/carboplatin } \\
\hline \multicolumn{4}{|c|}{$\S_{G N}=$ gemcitabine $/$ nedaplatine } \\
\hline${ }^{9} \mathrm{CDDP} / \mathrm{CPT}-11$ & & & \\
\hline
\end{tabular}

Postoperative outcomes are shown in Table 2. There were no differences in the total operation time, estimated blood loss, or intraoperative transfusion volumes. The time to cystectomy and time of ileal conduit urinary diversion were significantly shorter in the RARC + ICUD group than in the LRC + ECUD group. The number of dissected lymph nodes was significantly greater in the RARC + ICUD group than in the LRC + ECUD group. Among them, the number of lymph nodes with extended dissection (eLND) was significantly greater in the RARC + ICUD group than in the LRC + ECUD group. Regarding postoperative recovery, the median number of days to drinking and oral intake was significantly shorter in the RARC + ICUD group than in the LRC + ECUD group. The median length of hospital stay was 17 and 32 days in the RARC + ICUD and LRC + ECUD groups $(p<0.001)$, respectively. Based on blood sampling, the postoperative maximum value of $\mathrm{C}$-reactive protein was significantly lower in the RARC + ICUD group than in the LRC + ECUD group $(p=0.001)$. 
Table 2

Postoperative outcomes

\begin{tabular}{|c|c|c|c|}
\hline & $\begin{array}{l}\text { RARC + ICUD }(n= \\
\text { 26) }\end{array}$ & LRC + ECUD $(n=26)$ & $\begin{array}{l}\mathrm{P} \text { - } \\
\text { value }\end{array}$ \\
\hline Median total operation time, minutes (IQR) & $\begin{array}{l}516(470.75- \\
537.50)\end{array}$ & $\begin{array}{l}532.5(487.25- \\
579.25)\end{array}$ & 0.217 \\
\hline Median time to cystectomy & $\begin{array}{l}163(149.25- \\
181.50)\end{array}$ & $\begin{array}{l}194.5(160.25- \\
253.00)\end{array}$ & 0.007 \\
\hline Median time of lymph node dissection & $\begin{array}{l}84.5(60.25- \\
110.00)\end{array}$ & $92.0(68.50-112.50)$ & 0.862 \\
\hline $\begin{array}{l}\text { Median time of ileal conduit urinary } \\
\text { diversion }\end{array}$ & $\begin{array}{l}161.0(147.50- \\
180.25)\end{array}$ & $\begin{array}{l}201.5(186.25- \\
214.75)\end{array}$ & $<.001$ \\
\hline Median estimated blood loss, ml (IQR) & $\begin{array}{l}401(228.75- \\
590.00)\end{array}$ & $501(251.25-854.25)$ & 0.276 \\
\hline $\begin{array}{l}\text { Median intraoperative transfusion, } \mathrm{ml} \\
\text { (minimum to maximum) }\end{array}$ & $0(0-840)$ & $0(0-2800)$ & 0.758 \\
\hline Intraoperative complications & $0(0)$ & $1(3.8)$ & 1.000 \\
\hline Right common iliac vein injury & 0 & 1 & \\
\hline Type of lymph node dissection (\%) & & & 0.350 \\
\hline Extended & $25(96.2)$ & $22(84.6)$ & \\
\hline Standard & $0(0)$ & $1(3.8)$ & \\
\hline None & $1(3.8)$ & $3(11.5)$ & \\
\hline \multicolumn{4}{|l|}{$\begin{array}{l}\text { Median number of dissected lymph nodes } \\
\text { (IQR) }\end{array}$} \\
\hline All & $20.0(15-30)$ & $15(8-20)$ & 0.008 \\
\hline Extended & $20.5(17.25-30.25)$ & $16.5(11.0-20.75)$ & 0.015 \\
\hline Standard & none & 18 & \\
\hline \multicolumn{4}{|l|}{$\begin{array}{l}\text { Median number of positive lymph nodes } \\
\text { (minimum to maximum) }\end{array}$} \\
\hline All & $0(0-3)$ & $0(0-5)$ & 0.122 \\
\hline Extended & $0(0)$ & $0(0)$ & 0.163 \\
\hline Standard & none & 5 & \\
\hline
\end{tabular}

$\mathrm{IQR}=25 \%-75 \%$ interquartile range 


\begin{tabular}{|c|c|c|c|}
\hline & $\begin{array}{l}\text { RARC + ICUD }(n= \\
26)\end{array}$ & LRC + ECUD $(n=26)$ & $\begin{array}{l}\mathrm{P}- \\
\text { value }\end{array}$ \\
\hline Pathological T stage (\%) & & & 0.635 \\
\hline T0 & $4(15.4)$ & $5(19.2)$ & \\
\hline $\mathrm{Ta}$, Tis & $7(26.9)$ & $3(11.5)$ & \\
\hline T1 & $4(15.4)$ & $3(11.5)$ & \\
\hline T2 & $2(7.7)$ & $2(7.7)$ & \\
\hline T3 & $6(23.1)$ & $6(23.1)$ & \\
\hline T4 & $3(11.5)$ & $7(26.9)$ & \\
\hline Pathological N stage (\%) & & & 0.405 \\
\hline NO & $24(92.3)$ & $20(76.9)$ & \\
\hline N1 & $1(3.8)$ & $3(11.5)$ & \\
\hline N2 & $1(3.8)$ & $2(7.7)$ & \\
\hline N3 & $0(0.0)$ & $1(3.8)$ & \\
\hline Positive surgical margin (\%) & $1(3.8)$ & $5(19.2)$ & 0.191 \\
\hline Median days to walking, days (IQR) & $1(1-1.75)$ & $2(1-2)$ & 0.064 \\
\hline Median days to drinking, days (IQR) & $1(1-2)$ & $2(1.25-3)$ & 0.050 \\
\hline Median days to oral intake, days (IQR) & $3(3-5)$ & $5(4-5.75)$ & 0.003 \\
\hline Median length of hospital stay, days (IQR) & $17(15.00-20.75)$ & $32(28.25-36.75)$ & $\begin{array}{l}<.001 \\
0.001\end{array}$ \\
\hline \multicolumn{4}{|l|}{ Postoperative maximum value } \\
\hline Median CRP, mg/L (IQR) & $6.98(6.07-9.96)$ & $12.46(10.16-15.31)$ & 0.001 \\
\hline Median WBC, / $\mu \mathrm{L}$ (IQR) & $\begin{array}{l}11600(8950- \\
14125)\end{array}$ & $\begin{array}{l}11100(10025- \\
12650)\end{array}$ & 0.942 \\
\hline Median LDH, IU/L (IQR) & $203(177-226)$ & 207 (179.5-230.75) & 0.504 \\
\hline Median follow-up period, months (IQR) & $8(4-12.75)$ & $34(13.25-50.25)$ & $\begin{array}{l}< \\
0.001\end{array}$ \\
\hline IQR = 25\%-75\% interquartile range & & & \\
\hline
\end{tabular}

Postoperative complications are shown in Table 3. Although not significant, the RARC + ICUD group had fewer postoperative complications within 90 days than the LRC + ECUD group ( $23.1 \%$ vs. $42.3 \%, p=$ $0.237)$. There was no significant difference between the groups in the complication rates below or above 
Clavien-Dindo classification 3. Although no difference was observed, the RARC + ICUD group had a lower rate of ileus than the LRC + ECUD group ( $3.8 \%$ vs. $19.2 \%, p=0.191)$.

Table 3

Postoperative complications

\begin{tabular}{|llll|}
\hline & $\begin{array}{l}\text { RARC + ICUD ( } \mathbf{n}= \\
\text { 26) }\end{array}$ & LRC + ECUD ( $\mathbf{n = 2 6 )}$ & P-value \\
\hline $\begin{array}{l}\text { Postoperative complications, } \leq 90 \text { days } \\
(\%)\end{array}$ & $6(23.1)$ & $11(42.3)$ & 0.237 \\
\hline Clavien-Dindo classification, ver2.0 (\%) & & & 0.130 \\
\hline 1 & $1(3.8)$ & 0 & \\
\hline 2 & $4(15.4)$ & $7(26.9)$ & \\
\hline $3 a$ & 0 & $2(7.7)$ & \\
\hline $3 b$ & 0 & $2(7.7)$ & 0.743 \\
\hline $4 a$ & 0 & 0 & 0.350 \\
\hline $4 b$ & $1(3.8)$ & 0 & 0.191 \\
\hline$<3$ & $5(19.2)$ & $7(26.9)$ & 0.726 \\
\hline$\geq 3$ & $1(3.8)$ & $4(15.4)$ & 1.000 \\
\hline Ileus & $1(3.8)$ & $5(19.2)$ & $6(23.1)$ \\
\hline Infection & $4(15.4)$ & $1(3.8)$ & \\
\hline Ureteroileostomy stenosis & $2(7.7)$ & & \\
\hline
\end{tabular}

\section{Discussion}

ICUD is theoretically less invasive than ECUD, including reduced fluid loss from the intestines, reduced bleeding to prolong the pneumoperitoneum time, and shorter skin incisions and ureteral detachment areas.[6] However, when adapting ICUD, prolongation of the operation time, and damage to the ureter and intestinal tract by robotic arms are of concern. The number of studies evaluating the benefits of ICUD compared with ECUD is increasing, but no consensus has been reached.[3, 9, 10, 11]

In our analysis, although complication rates within 90 days were not significantly different, the RARC+ ICUD group had slightly better outcomes. The most common complications were infections in both groups. In the RARC + ICUD group, high-grade complications (Clavien-Dindo grade 3-5) were noted in the early stage of ICUD introduction. In a review of 26 institutions, although RARC + ICUD was associated with higher-grade complications than RARC + ECUD, they decreased with time.[6] Similarly, at our institution, there were few complications in the late stage of ICUD introduction and no serious complications developed in the latter stages in the RARC + ICUD group. 
In our study, we divided the operation time into time to cystectomy, time of lymph node dissection, and time of urinary diversion for procedure comparison because it is difficult to directly compare RARC + ICUD and LRC + ECUD. As a result, the time to cystectomy (median 163 vs. $194 \mathrm{~min}$ ) and time of urinary diversion with the ileal conduit (median 161 vs. $201.5 \mathrm{~min}$ ) were significantly shorter in the RARC + ICUD group. The total operation time (median 516 vs. $532.5 \mathrm{~min}$ ) and the time of lymph node dissection (median 84.5 vs. $92 \mathrm{~min}$ ) were equivalent between the groups.

There have been many comparisons between ICUD and ECUD. In the 83 previous studies, the mean operative time for the ileal conduit and neobladder by RARC + ICUD was 307 and 428 min, respectively, compared with 428 and 426 min by RARC + ECUD.[13] Furthermore, in a report on 10 institutions, the operative time for LRC + ICUD with an ileal conduit was approximately one hour longer (median 676 min vs. 616 min, $p=0.002$ ) than that for LRC + ECUD with an ileal conduit.[14] Thus, there were many reports on the operation time. In our study, there were no differences in the total operation time between the groups. However, by distinguishing the operation time for each part, we revealed that the operation time for cystectomy and urinary diversion with an ileal conduit by ICUD is shorter than that by ECUD.

In the initial stage of ICUD, there was a long operation time until the procedure was standardized, but once learned, ICUD with an ileal conduit was more stable than ECUD and shortened the operation time. In our experience, for ECUD with minimal incisions, especially in obese patients, it was difficult to pull the ureter out of the body and to anastomose due to the long distance between the suture position and the final position of the urinary tract. As a result, we needed longer incisions, which prolonged the operation time. On the other hand, ICUD enabled us to anastomose at the final position of the urinary tract and the skin incision was shorter, which made the operation more comfortable than ECUD with minimal incisions. As such, ICUD was performed in a shorter time than ECUD.

The difference in time to cystectomy reflected the difference in procedure between the robot and laparoscopy. RARC was previously associated with a lack of tactile feedback and longer operative time than LRC.[11] However, in a recent report, RARC had a similar operation time (median 326 vs. 315 min, $p$ $=0.279)$ to LRC.[12] The operational stability and robotic ergonomics of RARC may reduce the time to cystectomy.

Focusing on other secondary outcomes, length of hospital stay, number of days to walking, drinking and oral intake, and postoperative C-reactive protein in the RARC + ICUD group were significantly lower than those in the LRC + ECUD group. This suggested that the recovery time of the RARC + ICUD patients was superior to that of the LRC + ECUD patients. Thus, RARC + ICUD was less invasive than LRC + ECUD.

The number of eLND was significantly greater in the RARC + ICUD group than in the LRC + ECUD group. Zhang reported more eLND (17.8 \pm 5.7$)$ in the RARC group than standard LND (12.6 \pm 6.2$)$.[15] In this study, when performing eLND during RARC, we were able to extract more lymph nodes than when performing eLND during LRC. Therefore, RARC may enable more cranial lymph node dissections, especially common iliac and anterior sacral lymph nodes, than LRC. 
This study had two limitations. First, the number of patients was small. Although we had a small sample size, we demonstrated an advantage to introducing ICUD even in a facility where there were only approximately 20 cases of total cystectomy annually. Second, the follow-up period was relatively short. As the follow-up period was significantly shorter in the RARC + ICUD group, the long-term oncological outcomes between groups were unable to be compared.

\section{Conclusions}

RARC + ICUD had shorter cystectomy time and ileal conduit urinary diversion time than LRC + ECUD. This study suggested that RARC + ICUD is less invasive than LRC + ECUD and the recovery time of RARC + ICUD patients was superior to that of LRC + ECUD patients. Furthermore, we were able to extract more lymph nodes by eLND during RARC than during LRC. We consider ICUD with an ileal conduit following RARC to be the more advantageous procedure.

\section{Abbreviations}

ORC囚open radical cystectomy

LRC囚laparoscopic radical cystectomy

RARC『robot-assisted radical cystectomy

ICUD囚intracorporeal urinary diversion

ECUD: extracorporeal urinary diversion

\section{Declarations}

\section{Ethics approval and consent to participate}

This study was conducted according to the guidelines of the Declaration of Helsinki and approved by the ethics committee of Yokohama City University (protocol code, B201000042 and date of approval, November 10, 2020).

Written informed consent was waived because of the retrospective design.

\section{Consent for publication}

Not applicable.

\section{Availability of data and materials}

The datasets used and/or analysed during the current study are available from the corresponding author on reasonable request. 


\section{Competing interests}

The authors declare that they have no competing interests.

\section{Funding}

This research received no external funding.

\section{Authors' contributions}

Conceptualization, Atsuto Suzuki and Kazuhide Makiyama; Data curation, Atsuto Suzuki; Formal analysis, Atsuto Suzuki; Investigation, Atsuto Suzuki; Methodology, Kazuhide Makiyama; Project administration, Kazuhide Makiyama; Resources, Kazuhide Makiyama, Kentaro Muraoka, Tomoyuki Tatenuma, Kimito Osaka, Yumiko Yokomizo, Narihiko Hayashi, Hisashi Hasumi and Yusuke Ito; Software, Atsuto Suzuki; Supervision, Keichi Kondo and Noboru Nakaigawa; Validation, Atsuto Suzuki; Visualization, Atsuto Suzuki; Writing - original draft, Atsuto Suzuki; Writing - review \& editing, Atsuto Suzuki and Kazuhide Makiyama.

\section{Acknowledgments}

The authors thank Medical English Service (www.med-english.com) for the English language review.

\section{References}

1. Stein JP, Lieskovsky G, Cote R, Groshen S, Feng AC, Boyd S, et al. Radical cystectomy in the treatment of invasive bladder cancer: long-term results in 1,054 patients. J Clin Oncol.

2. Lin T, Fan X, Zhang C, Xu K, Liu H, Zhang J, et al. A prospective randomised controlled trial of laparoscopic vs open radical cystectomy for bladder cancer: perioperative and oncologic outcomes with 5-year follow-upT Lin et al. Br J Cancer. 2014;110: 842-9.

3. Ahmed K, Khan SA, Hayn MH, Agarwal PK, Badani KK, Balbay MD, et al. Analysis of intracorporeal compared with extracorporeal urinary diversion after robot-assisted radical cystectomy: results from the International Robotic Cystectomy Consortium. Eur Urol. 2014;65: 340-7.

4. Challacombe BJ, Bochner BH, Dasgupta P, Gill I, Guru K, Herr H, et al. The role of laparoscopic and robotic cystectomy in the management of muscle-invasive bladder cancer with special emphasis on cancer control and complications. Eur Urol. 2011;60: 767-75.

5. Hrouda D, Adeyoju AA, Gill IS. Laparoscopic radical cystectomy and urinary diversion: fad or future? BJU Int. 2004;94: 501-5.

6. Brassetti A, Cacciamani G, Anceschi U, Ferriero M, Tuderti G, Miranda G, et al. Long-term oncologic outcomes of robot-assisted radical cystectomy (RARC) with totally intracorporeal urinary diversion (ICUD): a multi-center study. World J Urol. 2020;38: 837-43.

7. BRICKER EM. Bladder substitution after pelvic evisceration. Surg Clin North Am. 1950;30: 1511-21. 
8. Studer UE. Bladder substitution with an ileal low-pressure reservoir. Prog Clin Biol Res. 1989;303: 803-9.

9. Kanda Y. Investigation of the freely available easy-to-use software 'EZR' for medical statistics. Bone Marrow Transplant. 2013;48: 452-8.

10. Zhang JJH, Ericson KJ, Thomas LJ, Knorr J, Khanna A, Crane A, et al. Large Single-Institution Comparison of Perioperative Outcomes and Complications in Open Radical Cystectomy, Intracorporeal Robot-Assisted Radical Cystectomy, and Robotic Extracorporeal Approach. J Urol. 2019;203: 512-521.

11. Hussein AA, May PR, Jing Z, Ahmed YE, Wijburg CJ, Canda AE, et al. Outcomes of Intracorporeal Urinary Diversion after Robot-Assisted Radical Cystectomy: Results from the International Robotic Cystectomy Consortium. J Urol. 2018;199: 1302-11.

12. Su S, Gu L, Ma X, Li H, Wang B, Shi T, et al. Comparison of Laparoscopic and Robot-assisted Radical Cystectomy for Bladder Cancer: Perioperative and Oncologic Outcomes. Clin Genitourin Cancer. 2019;17: e1048-e53.

13. Tanneru K, Jazayeri SB, Kumar J, Alam MU, Norez D, Nguyen S, et al. Intracorporeal versus extracorporeal urinary diversion following robot-assisted radical cystectomy: a meta-analysis, cumulative analysis, and systematic review. J Robot Surg. 2020.

14. Kanno T, Inoue T, Kawakita M, Ito K, Okumura K, Yamada H, et al. Perioperative and oncological outcomes of laparoscopic radical cystectomy with intracorporeal versus extracorporeal ileal conduit: A matched-pair comparison in a multicenter cohort in Japan. International Journal of Urology. 2020;27: 559-565.

15. Zhang S, Lin T, Zhang Q, Zhang S, Liu G, Ji C, et al. Comparison of perioperative outcomes in robotassisted radical cystectomy and laparoscopic radical cystectomy. Int J Med Robot. 2020;16: e2074. 